\title{
A comparative study on the efficacy of levofloxacin and ceftazidime in acute exacerbation of bronchiectasis
}

\author{
K.W.T. Tsang, W-M. Chan, P-L. Ho*, K. Chan, W-K. Lam, M.S.M. Ip
}

A comparative study on the efficacy of levofloxacin and ceftazidime in acute exacerbation of bronchiectasis. K.W.T. Tsang, W.M. Chan, P-L. Ho, K. Chan, W-K. Lam, M.S.M. Ip. (C) ERS Journals Ltd 1999.

ABSTRACT: A prospective randomized study was performed in order to compare the efficacy of oral levofloxacin, a new $S$ - isomer of ofloxacin, with intravenous ceftazidime in the empirical treatment of acute exacerbations of bronchiectasis.

Consecutive patients with acute exacerbation of bronchiectasis were recruited at a tertiary referral centre and were randomized to receive 10 days' treatment with either oral levofloxacin ( $300 \mathrm{mg}$ b.i.d.) or ceftazidime (1 g i.v. t.i.d.). Body temperature, cough score, dyspnoea score, sputum purulence and volume and white blood cell and neutrophil count were assessed on day 1 and day 10 .

Thirty-five patients (mean age 61 yrs, 15 males) completed the study; 17 of these were in the levofloxacin group. There was no significant difference in the distribution of sputum pathogens or clinical parameters between the two groups at entry to and completion of the study. Both groups of patients showed significant improvement in 24-h sputum volume, sputum purulence score, cough score and dyspnoea score $(p<0.001)$ but there was no significant difference between these two groups at entry to or on completion of the study $(p>0.05)$.

The results of this study suggest that oral administration of levofloxacin is as effective as parenteral ceftazidime in the empirical treatment of exacerbations in bronchiectasis.

Eur Respir J 1999; 14: 1206-1209.
University Depts of Medicine and *Microbiology, The University of Hong Kong, Queen Mary Hospital, Hong Kong.

Correspondence: M.S.M. Ip, Division of Respiratory and Critical Care Medicine, University Dept of Medicine, Queen Mary Hospital, The University of Hong Kong, Pokfulam, Hong Kong SAR, China, Fax: 85228725828

Keywords: Bronchiectasis ceftazidime

exacerbations

levofloxacin

Received: December 171998

Accepted after revision June 291999

This study was partially sponsored by Daiichi Pharmaceutical Asia
Bronchiectasis is a common respiratory disorder amongst the Chinese and affected patients suffer from chronic sputum production, recurrent exacerbations and, in some cases, progressive lung destruction. Whilst the pathogenesis of bronchiectasis clearly includes infective and inflammatory elements [1] and it has recently been shown that inhaled fluticasone therapy is effective in reducing airway inflammation [2], antibiotic therapy remains the mainstay of treatment in exacerbations. Pseudomonas aeruginosa constituted $25-50 \%$ of sputum bacterial isolates in Hong Kong [3], rendering most $\beta$-lactam antibiotics theoretically ineffective. Standard agents directed against Pseudomonas, such as ceftazidime, aminoglycosides and imipenem, however, require parenteral administration. Ceftazidime is a broad-spectrum third-generation cephalosporin, which is effective in exacerbations of cystic fibrosis (CF) and non-CF bronchiectasis [4] and is active against $P$. aeruginosa isolates in vitro [5]. Levofloxacin is a new oral fluoroquinolone, which is active against many respiratory pathogens including Streptococcus pneumoniae, Staphylococcus aureus, Branhamella catarrhalis, Haemophilus influenzae and P. aeruginosa [5, 6]. A randomized prospective study was performed in order to compare the efficacy of oral levofloxacin with that of intravenous ceftazidime in the treatment of acute exacerbations of bronchiectasis.

\section{Materials and methods}

\section{Patient recruitment and assessment}

Consecutive patients admitted to Queen Mary Hospital for treatment of acute exacerbation of bronchiectasis were recruited with written consent, in a block-randomized prospective fashion, between June 1996 and December 1997 to receive either intravenous ceftazidime (1 g t.i.d.) or oral levofloxacin (300 $\mathrm{mg}$ b.i.d.) for 10 days. Inclusion criteria included: age $\geq 21 \mathrm{yrs}$, high-resolution computed tomography (HRCT)-proven bronchiectasis, and evidence of exacerbation defined as a "subjective and persistent $(\geq 24 \mathrm{~h})$ deterioration in at least 3 respiratory symptoms including cough, dyspnoea, haemoptysis, increased sputum purulence or volume, and chest pain; with or without fever $\left(\geq 37.5^{\circ} \mathrm{C}\right)$, radiographic deterioration, systemic disturbances, or deterioration in chest signs including crackles and dullness on auscultation and percussion respectively [2]". Exclusion criteria included: known allergy to cephalosporins or quinolones, antimicrobial treatment within $72 \mathrm{~h}$ prior to treatment immunosuppression due to drug treatment and/or diseases (e.g. haemic malignancies) and the presence of other unstable nonrespiratory diseases.

Patients were followed-up until they reached their steady state after treatment (defined as no significant changes 
in respiratory symptoms and signs and sputum volume for $>3$ weeks without any alteration in medications or spirometry results ((forced expiratory volume in one second (FEV1) and forced vital capacity (FVC))). The number of exacerbations in the preceding 12 months was determined for each patient by meticulous history taking and clinical chart review. The number of bronchiectatic lung lobes was determined by using thin-section HRCT within 12 months before the study [7]. The aetiology of bronchiectasis was determined after history taking, examination and investigations including nasal respiratory ciliary beat frequency assessment [8].

\section{Clinical assessment}

Clinical response was assessed daily by a research physician (W-M. Chan) who was blind to the treatment protocol, between 10:00 and 11:00 on day 1 and on day 10, before the morning session of the twice-daily physiotherapy sessions. Patients were asked to score severity of cough as $0,1,2,3$ or 4 corresponding to no, very mild, mild, moderate and severe cough respectively, using a visual analogue chart [3]. Dyspnoea score was assessed similarly [3]. Patients were withdrawn if there was failure to improve clinically (defined as no change in the clinical or sputum score compared with day 1) or deterioration (defined as deterioration in original respiratory symptom or sputum scores, or the emergence of new respiratory symptoms).

\section{Sputum assessment}

The volume of a 24-h sputum specimen was determined as described previously [2]. Sputum purulence score was determined as the highest score from three random aliquots selected from the centre of a 24-h specimen, and expressed as $0,1,2,3,4,5$ and 6 corresponding to no, transparent, opaque and milky white, grey, pale green, moderately green and dark-green sputum respectively [9]. After the clinical assessment, as described above, patients received chest physiotherapy (expectoration-aiding manoeuvres performed until no further sputum was obtained) before fresh sputum was collected by the research physician. Standard microbiological procedures were performed on day 1 and on day 10 (except when there was no sputum), to identify all of the sputum bacteria and classify them into pathogenic ( $P$. aeruginosa, $H$. influenzae, $S$. pneumoniae, Staphylococcus aureus and Mycobacteria) and nonpathogenic bacteria (Neisseria, $\alpha$-haemolytic streptococci, diphtheroids and coagulase-negative staphylococci) [3]. The sensitivity of the isolates was determined by means of the disc diffusion method and interpreted according to the National Committee for Clinical Laboratory Standards [10].

\section{Statistical analysis}

Study size estimation was performed using changes in 24-h sputum volume as the main outcome parameter. Assuming that a two-fold change in sputum volume was of clinical significance, and assuming a type I error of 0.05 and accepting a type II error of 0.2 (power of 0.8 ), a minimum of 17 subjects was needed in each group. The clinical parameters of the patients on day 1 and on day 10 were compared using the Statistical Analysis Software system, between the two study groups using unpaired ttests and Wilcoxon rank sum tests for continuous and ordinal data respectively. A p-value $<0.05$ was taken as significant.

\section{Results}

\section{Patient demography and clinical features}

The demographic and clinical features of the patients are shown in table 1. Between June 1996 and December 1997, 37 patients were recruited and 35 completed the study. Eighteen and 17 patients received ceftazidime and levofloxacin respectively. One patient was withdrawn from the ceftazidime group (day 3) and one from the levofloxacin group (day 6) due to clinical deterioration and severe haemoptysis respectively. Among patients who were not withdrawn, three in the ceftazidime group, reported mild symptoms, namely dizziness and dyspepsia, and four in the levofloxacin group, namely dizziness, insomnia and mild skin rash. There was no significant difference in age, sex distribution, steady-state spirometry results, number of lobes affected by bronchiectasis or exacerbation frequency between the two treatment groups ( $p>0.05)$. The aetiology of bronchiectasis was determined for the ceftazidime and levofloxacin groups as idiopathic $(\mathrm{n}=11$ and 10 respectively), post-tuberculous ( $\mathrm{n}=4$ and 5 respectively) and post-pneumonic ( $\mathrm{n}=3$ and 2 respectively).

\section{Sputum and clinical assessment}

There was no significant difference in all clinical parameters between the ceftazidime and levofloxacin group on entry to the study (tables 1 and 2). Patients in both groups improved, with falls in body temperature, white blood cell count, neutrophil count, sputum parameters and symptom scores. There was no difference in the

Table 1. - Demographic and clinical features of patients on entry into the study

\begin{tabular}{lcc}
\hline & $\begin{array}{c}\text { Ceftazidime } \\
\text { group }\end{array}$ & $\begin{array}{c}\text { Levofloxacin } \\
\text { group }\end{array}$ \\
\hline Subjects n & 18 & 17 \\
Age yrs & $59 \pm 15$ & $63 \pm 9$ \\
Sex F/M & $12 / 6$ & $8 / 8$ \\
Steady-state of FEV1 L & $0.95 \pm 0.42$ & $0.89 \pm 0.41$ \\
& $49 \pm 22$ & $41 \pm 16$ \\
Steady-state FVC pred $\quad$ L & $1.57 \pm 0.61$ & $1.54 \pm 0.61$ \\
& $58 \pm 17.81$ & $52.56 \pm 17.46$ \\
Exacerbations n-yr ${ }^{-1}$ \% pred & $3.1 \pm 2.8$ & $2.1 \pm 1.6$ \\
Lung lobes affected by bron- & $3.2 \pm 1.7$ & $3.7 \pm 1.2$ \\
chiectasis n & & \\
Current medication n & & \\
Inhaled bronchodilators & 11 & 15 \\
Nebulized aminoglycosides & 4 & 2 \\
Inhaled steroid & 6 & 4 \\
Aminophylline & 2 & 2 \\
Diuretic & 2 & 2 \\
\hline
\end{tabular}

Data are presented as mean \pm SD. F: female; M: male; FEV1: forced expiratory volume in one second; FVC: forced vital capacity. There was no significant difference between the two groups $(\mathrm{p}<0.05)$. 
Table 2. - Clinical response in patients on entry and on completion of treatment

\begin{tabular}{|c|c|c|c|c|c|c|c|c|}
\hline & \multicolumn{3}{|c|}{ Ceftazidime group } & \multicolumn{3}{|c|}{ Levofloxacin group } & \multirow[b]{2}{*}{ p-value } & \multirow[b]{2}{*}{$p$-value ${ }^{\S}$} \\
\hline & Day 1 & Day 10 & p-value* & Day 1 & Day 10 & p-value ${ }^{+}$ & & \\
\hline \multirow{3}{*}{$\begin{array}{l}\text { Body temperature }{ }^{\circ} \mathrm{C} \\
\text { Total white cell count } \\
10^{9} \text { cells } \cdot \mathrm{dL}^{-1}\end{array}$} & $37.5 \pm 0.6$ & $36.8 \pm 0.4$ & 0.0006 & $37.4 \pm 0.8$ & $36.6 \pm 0.4$ & 0.02 & 0.90 & 0.17 \\
\hline & & & & & & & & \\
\hline & $11.2 \pm 3.4$ & $7.0 \pm 1.7$ & 0.0001 & $10.7 \pm 4.0$ & $7.8 \pm 2.4$ & 0.01 & 0.95 & 0.28 \\
\hline \multicolumn{9}{|l|}{ Neutrophil count } \\
\hline $10^{9}$ cells $\cdot \mathrm{dL}^{-1}$ & $8.7 \pm 2.9$ & $4.6 \pm 1.5$ & 0.0001 & $7.7 \pm 3.4$ & $5.3 \pm 2.5$ & 0.02 & 0.73 & 0.40 \\
\hline 24-h sputum volume $\mathrm{mL}$ & $60(40-80)$ & $20(5-30)$ & 0.0002 & $72.5(35-135)$ & $16(10-50)$ & 0.002 & 0.64 & 0.53 \\
\hline Sputum purulence score $0-6$ & $4(3-5)$ & $1(1-3)$ & 0.0007 & $3(3-5)$ & $1.5(1-3)$ & 0.03 & 0.17 & 0.44 \\
\hline Cough score $0-4$ & $3(1-3)$ & $1(1-1)$ & 0.0005 & $2(1-3)$ & $1(1-1)$ & 0.002 & 0.97 & 0.37 \\
\hline Dyspnoea score $0-4$ & $1(1-3)$ & $0.5(0-1)$ & 0.001 & $2(1-3)$ & $1(0-1)$ & 0.002 & 0.27 & 0.48 \\
\hline \multicolumn{9}{|l|}{ Sputum bacterial isolates $n$} \\
\hline Pseudomonas aeruginosa & 6 & 4 & & 4 & 2 & & & \\
\hline Haemophilus influenzae & 3 & 0 & & 1 & 0 & & & \\
\hline Acinetobacter & 1 & 0 & & 0 & 0 & & & \\
\hline Mycobacterium chelonei & 1 & 0 & & 0 & 0 & & & \\
\hline Branhamella catarrhalis & 1 & 0 & & 1 & 0 & & & \\
\hline Staphylococcus aureus & 0 & 1 & & 1 & 2 & & & \\
\hline Streptococcus pneumoniae & 1 & 0 & & 0 & 0 & & & \\
\hline Xanthomonas pneumoniae & 0 & 0 & & 0 & 1 & & & \\
\hline Commensals & 5 & 13 & & 9 & 11 & & & \\
\hline
\end{tabular}

Data are presented as median (interquartile range), mean \pm SD or $n .{ }^{*},{ }^{+}$: day 1 versus day 10 for ceftazidime and levofloxacin groups respectively using paired t-tests and Wilcoxon sign-rank tests as appropriate; ${ }^{\#, ~}$ : ceftazidime versus levofloxacin at day 1 and day 10 respectively using unpaired t-test and Wilcoxon rank-sum tests as appropriate.

treatment effects between the two groups in clinical and sputum parameters on study completion. P. aeruginosa and $H$. influenzae were the most frequently isolated sputum pathogens. One pathogen in each of the two groups was resistant to the respective antibiotics (Mycobacterium chelonei in the ceftazidime and $P$. aeruginosa in the levofloxacin group). Eradication of respiratory pathogens from sputum was achieved in 69 and $57 \%$ of ceftazidime- and levofloxacin-treated cases. The clinical response to treatment was similar between the two treatment arms (table 2).

\section{Discussion}

The results of this prospective randomized study show that, in patients who had acute exacerbations, improvements in body temperature, 24-h sputum volume, sputum purulence, cough and dyspnoea were similar with empirical intravenous ceftazidime and oral levofloxacin therapy (table 2). P. aeruginosa and H. influenzae were the commonest pathogens isolated in sputum on entry into the study (table 2) $[3,11]$. Eradication of respiratory pathogens from sputum was achieved in 69 and $57 \%$ of ceftazidime- and levofloxacin-treated cases. As reported in previous studies, a substantial proportion of the patients $(41 \%)$ did not have identifiable sputum bacterial pathogens $[3,11]$.

Most intratracheobronchial bacteria are associated with respiratory mucus rather than with the mucosa $[12,13]$. The ratios between the levels of penicillins, cephalosporins, aminoglycosides, tetracycline, macrolides and quinolones in respiratory secretions (sputum) to serum, which are measures of antibiotic penetration into the target site, are $2-6,15-25,20-30,10-30,10$ and $90-100 \%$ respectively [14]. As antibiotics generally penetrate poorly into respiratory secretions, many bacteria in the respiratory tract are likely to be exposed to less than the minimal inhibitory concentration (MIC) of antibiotics during treatment of an infective exacerbation. Although sub-MIC antibiotics protect respiratory epithelium in its interaction with respiratory pathogens such as $H$. influenzae [13] and $P$. aeruginosa [15], it is conventional wisdom to try to achieve supra-MIC at the target sites.

The empirical use of antibiotics, pending results of sputum culture, is commonly practised in the treatment of exacerbations in bronchiectasis. This choice of antibiotics is usually tailored to the patient and with consideration of local knowledge on the likely pathogens causing exacerbations in bronchiectasis. Sputum microbiology is well defined in bronchiectatic and CF patients in the West with $H$. influenzae one of the commonest bacteria to be isolated from sputum followed by $S$. pneumoniae [11] although $B$. catarrhalis [16], viruses [17] and, particularly, P. aeruginosa are also responsible in many cases [3]. Consequently, $\beta$-lactams including amoxycillin/clavulanic acid [18]; cephalosporins including loracarbef [19] and cefuroxime [20], quinolones including ofloxacin [3]: and co-trimoxazole [21] have been used either alone or in combination in these situations. Ceftazidime is useful even as monotherapy in the treatment of pulmonary infections in CF [4].

A recent systematic study on 100-steady state bronchiectatic patients showed that Pseudomonas aeruginosa and Haemophilus influenzae were the pathogens isolated in the sputum of 33 and $10 \%$ of cases in Hong Kong [22]. This suggests that antibiotics active against Pseudomonas aeruginosa and Haemophilus influenzae should be considered as the initial empirical treatment pending the results of sputum microbiological assessment in the authors' locality. Effective agents therefore include ceftazidime, aminoglycosides, imipenem and quinolones. The quinolones have distinct advantages over the former three families as they are available in oral preparation and demonstrate excellent penetration into tissue and particularly bronchial secretions [6]. 


\section{References}

1. Cole PJ. Inflammation: a two edged-sword the model of bronchiectasis. Eur J Respir Dis 1936; 147 Suppl: 615.

2. Tsang KWT, Ho PL, Lam WK, et al. Inhaled fluticasone reduces sputum inflammatory indices in severe bronchiectasis. Am J Respir Crit Med 1998; 158: 723-727.

3. Lam WK, Chau PY, So SY, et al. Ofloxacin compared with amoxycillin in treating infective exacerbations in bronchiectasis. Resp Med 1989; 83: 299-303.

4. Padoan R, Cambisano W, Costantini D, et al. Ceftazidime monotherapy versus combined therapy in Pseudomonas infections in cystic fibrosis. Pediatr Infect Dis 1937; 6: 648-653.

5. Jones RN, Kehrberg EN, Erwin ME, et al. Prevalence of important pathogens and antimicrobial activity of parenteral drugs at numerous medical centers in the United States, I. Study on the threat or emerging resistance: real or perceived? Fluoroquinolone Resistance Surveillance Group. Diagn Microbiol Infect Dis 1994 19: 203-215.

6. Davis R, Bryson HM. Levofloxacin. A review of its antibacterial activity, pharmaco-kinetics and therapeutic efficacy. Drugs 1994; 47: 677-700.

7. McGuniness G, Naidich DP, Leitman BS, McCauley DI. Bronchiectasis: CT evaluation. Am J Radiol 1993; 160: 253-259.

8. Tsang KWT, Ooi C, Ip MS, et al. Diffuse panbronchiolitis in Chinese patients. Thorax 1998; 53: 274-280.

9. Deneuville E, Perrot-Minot C, Pennaforte F, et al. Revisited physiochemical and transport properties of respiratory mucus in genotyped cystic fibrosis patients. $\mathrm{Am} \mathrm{J}$ Respir Crit Care Med 1997; 156: 166-172.

10. National Committee for Clinical Laboratory Standards. Performance standards antimicrobial disc susceptibility tests - Fifth Edition: Approved Standard M2-A5. NCCLS, Villanova, PA, 1993.

11. Hill SL, Morrison HM, Burnett D, et al. Short term response of patients with bronchiectasis to treatment with amoxycillin given in standard or high doses orally and by inhalation. Thorax 1986; 41: 559-565.
12. Tsang KWT, Rutman A, Kanthakumar K, et al. Haemophilus influenzae infection of human respiratory mucosa in low concentrations of antibiotics. Am Rev Respir Dis 1993; 148: 201-207.

13. Tsang KW, Rutman A, Dewar A, et al. Interaction of Pseudomonas aeruginosa with human respiratory mucosa in vitro. Eur Respir J 1994; 7: 1746-1753.

14. Valcke Y, Pauwels R, Van der Straeten M. Pharmacokinetics of antibiotics in the lungs. Eur Respir J 1990; 3: 715-722.

15. Tanaka E, Kathakumar K, Cundell D, et al. The effects of erythromycin on ciliotoxin production by Pseudomonas aeruginosa in vitro. J Antimicrob Chemother 1994; 33: 765-775.

16. Klingman KL, Pye A, Murphy TF, et al. Dynamics of respiratory tract colonization by Branhamella catarrhalis in bronchiectasis. Am J Respir Crit Med 1995; 152: 10721078.

17. Wiselka MJ, Kent J, Cookson JB, et al. Impact of respiratory virus infection in patients with chronic chest disease. Epidemiol Infect 1993; 111: 337-346.

18. Stockley RA, Dragicevic P, Burnett D, et al. Role of betalactamases in the response of pulmonary infections to amoxycillin/clavulanate. J Antimicrob Chemother 1999; 24 (suppl B): 73-81.

19. Hill SL, Bilton D, Johnson M, et al. Sputum and serum pharmacokinetics of loracarbef (LY 163892) in patients with chronic bronchial sepsis. J Antimicrob Chemother 1995; 36: 446-447.

20. Brambilla $\mathrm{C}$, Kastanakis $\mathrm{S}$, Knight $\mathrm{S}$, et al. Cefuroxime and cefuroxime axetil versus amoxicillin plus clavulanic acid in the treatment of lower respiratory tract infections. Eur J Clin Microbiol Infect Dis 1992; 11: 118-124.

21. Honda T, Hayasaka M, Hachiya T, et al. Two cases of severe bronchiectasis successfully treated with a prolonged course of trimethoprim/sulfamethoxazole. Intern Med 1996; 35: 979-983.

22. Ho PL, Lam WK, Ip MSM, Chan KN, Yuen KY, Tsang KWT. The effects of Pseudomonas aeruginosa infection in clinical parameters in steady state bronchiectasis. Chest 1998; 114: 1623-1629. 